\title{
YAP1 inhibits ovarian endometriosis stromal cell invasion through ESR2
}

\author{
Cheng Zeng*, Pei-Li Wu*, Zhao-Tong Dong, Xin Li, Ying-Fang Zhou and Qing Xue \\ Department of Obstetrics and Gynecology, Peking University First Hospital, Beijing, China \\ Correspondence should be addressed to Q Xue; Email: xueqingqq@hotmail.com
}

*(C Zeng and P-L Wu contributed equally to this work)

\begin{abstract}
Endometriosis is an estrogen-dependent disease, and estrogen receptor 2 (ESR2) plays a critical role in the pathogenesis of ovarian endometriosis by promoting cell invasion. Yes-associated protein 1 (YAP1) plays suppressive roles in several types of tumors. However, the relationship between YAP1 and ESR2 is not fully understood. The aim of this study was to investigate the regulatory mechanism of YAP1 in terms of ESR2 and YAP1 regulation of endometriotic stromal cell (ECSC) invasion in ovarian endometriosis. Our results demonstrated that YAP1 mRNA and protein levels in eutopic endometrium (EU) tissues were higher than those in paired ectopic endometrium (EC) tissues. ECSCs transfected with siYAP1 exhibited a significant increase in both ESR2 mRNA levels and protein expression. Simultaneously, YAP1 overexpression in ECSCs yielded the opposite results. Co-IP assays demonstrated YAP1-NuRD complex formation by YAP1, CHD4 and MTA1 in ECSCs. YAP1 bound to two sites, $(-539,-533)$ and $(-158,-152)$, upstream of the ESR2 transcription initiation site. YAP1 binding to the two sites of the ESR2 promoter in ECSCs was significantly lower than that in eutopic endometrial stromal cells (EUSCs) from EU tissues. ECSCs transfected with siYAP1 exhibited increased invasion activity, while ECSCs transfected with siESR2 showed inhibition of invasion. However, transfection with siYAP1 and siESR2 together decreased the number of invading cells compared with transfection with siYAP1 alone. Therefore, we conclude that decreased levels of YAP1 in ovarian endometriomas enhance ESR2 expression via formation of a YAP1-NuRD complex, which further binds to the ESR2 promoters. Furthermore, YAP1 inhibits ECSCs invasion.

Reproduction (2020) 160 481-490
\end{abstract}

\section{Introduction}

Endometriosis is an estrogen-dependent disease (Bulun 2009) affecting up to $10 \%$ of women of reproductive age (Ryan \& Taylor 1997). Hormonal treatment or conservative surgery achieves significant pain relief in only approximately $50 \%$ of women diagnosed with endometriosis, and the symptom recurrence rate is high. Thus, novel and effective therapies for endometriosis are urgently needed (Vercellini et al. 2014, Greene et al. 2016, Liu et al. 2017). Estradiol is a key hormone for the growth and persistence of endometriotic tissue (Maia et al. 2012). Ovarian endometriotic lesions show high estradiol biosynthesis compared with the normal endometrium (Jarzabek et al. 2013). However, the effects of estrogen are mediated primarily via nuclear estrogen receptors, including ER $\alpha$ and $E R \beta$, which are encoded by ESR1 and ESR2, respectively (Jarzabek et al. 2013). Previous studies have suggested that estrogen receptor 2 (ESR2)-selective agonists might be therapeutic in a rodent endometriosis model (Xiu-Li et al. 2009) and that ESR2 interacts with the inflammasome complex and cytoplasmic apoptotic machinery to enhance the proliferative and adhesive activities of endometriotic tissues and prevent tumor necrosis factor $\alpha$ (TNF- $\alpha$ ) induced cell death (Han et al. 2015), indicating a key role for ESR2 in endometrial and endometriosis growth regulation.

Yes-associated protein 1 (YAP1) is a critical component of the size-controlling Hippo signaling pathway (Pan 2010). Through a kinase cascade, this pathway targets YAP1 for phosphorylation, preventing its translocation to the nucleus, where it functions as a transcriptional coactivator. As a transcriptional coactivator, YAP1 can bind to oncogenes or tumor suppressor gene transcription factors, the most important of which are the TEAD transcription factors. A YAP1/TAZ-TEAD complex recruits the nucleosome remodeling and histone deacetylase (NuRD) complex to deacetylate histones and alters nucleosome occupancy at target genes (Kim et al. 2015). The current dogma suggests that restriction of YAP1 transcriptional activity is the principal mechanism of growth and tumor suppression by the Hippo pathway (Mo et al. 2014). Indeed, nuclear YAP1 is a powerful mediator of organ growth, cancer cell proliferation and invasion, and tumor growth (Camargo et al. 2007, Dong et al. 2007). The role of YAP1 in cancer 
development remains controversial (Zanconato et al. 2016). YAP1 was originally found to promote cell proliferation and transformation. YAP1 overexpression has been linked to tumor progression and worse survival in the context of certain malignancies. However, YAP1 has been recently recognized as a tumor suppressor gene because it also induces apoptosis. Therefore, the expression and function of YAP1 in specific diseases require further investigation.

Currently, few studies have considered the function of YAP1 in endometriosis, and the relationship between YAP1 and ESR2 in ovarian endometriosis is still not well understood. Because ESR2 has been shown to regulate the invasion of ectopic endometrial cells in ovarian endometriosis, whether YAP1 suppresses cell invasion activity by regulating ESR2 is worth exploring. Thus, the aim of this study was to investigate the regulatory mechanism of YAP1 in terms of ESR2 and YAP1 regulation of endometriotic stromal cell invasion in ovarian endometriosis. We propose that YAP1 inhibits ESR2 expression by participating in NuRD transcriptional repression complexes and that YAP1 inhibits MMP2 and MMP9 expression by inhibiting ESR2 expression.

\section{Materials and methods \\ Participants and primary cell culture}

Ectopic endometrium (EC) tissues from the cyst walls of ovarian endometriomas and eutopic endometrium (EU) tissues were obtained from 25 women with endometriosis immediately after surgery, resulting in 25 self-controlled pairs. All patients (age range: 23-40 years) had regular menstrual cycles and none received hormonal therapy for at least 3 months before surgery. The diagnoses for all samples were histologically confirmed. The experimental protocol was approved by the Institutional Review Board of Peking University (No. 2014 (789)), and an informed consent form was signed by each patient before the samples were used. Human endometriotic or ectopic stromal cells (ECSCs) from ovarian endometriomas and eutopic endometrial stromal cells (EUSCs) were isolated from 17 tissue samples using the protocol previously described by Ryan et al. with minor modifications (Ryan et al. 1994). Briefly, endometriotic or endometrial tissues were digested with collagenase $(1 \mathrm{mg} / \mathrm{mL}$; Sigma-Aldrich) and deoxyribonuclease I $(0.1 \mathrm{mg} / \mathrm{mL}$; SigmaAldrich). Epithelial cells were removed by filtration of the cell suspension through a $75-\mu \mathrm{m}$ sieve. Stromal cells were then harvested and cultured to confluence in DMEM/F-12 containing $10 \%$ fetal bovine serum (Gibco, Thermo Fisher Scientific).

\section{RNA extraction and real-time polymerase chain reaction}

Total RNA was extracted from cultured primary stromal cells using TRIzol reagent (Life Technologies, Thermo Fisher Scientific) and quantified with a NanoDrop 1000 spectrophotometer (Thermo Fisher Scientific). Two micrograms of total RNA was used to synthesize cDNA using High-Capacity
cDNA RT kits (4368814, Life Technologies, Thermo Fisher Scientific) according to the manufacturer's instructions. Realtime (RT) quantitative PCR (qPCR) was performed using an ABI 7500 Sequence Detection system and an ABI Power SYBR-Green gene expression system (4367660, Applied Biosystems, Thermo Fisher Scientific) to quantify YAP1, ESR2, MMP2 and MMP9 mRNA levels. The following primers were used: YAP1: (F) 5'-ACCCCAGATGACTTCCTGAACA-3', (R) 5'-GCAGGGTGCTTTGGTTGATAG-3'; ESR2: (F) 5'-ATGATCAGCTGGGCCAAGAA-3', (R) 5'-CCACATCAGCCC-CATCATTAA-3'; MMP2: (F) 5'-CCCACTGCGGTTTTCTCGAAT-3', (R) 5' CAA-AGGGGTATCCATCGCCAT-3'; MMP9: (F) 5'-AGA-CCTGGGCAGATTCCAAAC-3', (R) 5'-CGGCAAGTCTTCCGAGTAGT-3'; and 185 rRNA: (F) 5'-AGGAATTCCCAGTAAGTGCG-3', (R) 5'-GCCTCACTAAACCATCCAA-3'. Human $18 \mathrm{~S}$ rRNA was used as an internal control. The relative quantification of all transcripts was analyzed by the comparative threshold cycle method.

\section{Western blot analysis}

ECSCs were washed in ice-cold PBS and lysed in mammalian protein extraction reagent (KeyGen Biotech, China) containing a protease inhibitor cocktail (Amresco, USA). Lysates were centrifuged at $17,949 \mathrm{~g}$ for $20 \mathrm{~min}$ at $4^{\circ} \mathrm{C}$. Protein concentrations were determined using a micro-BCA protein assay kit (KeyGen, Biotech). Equal amounts of total protein were resolved on 8 or $10 \%$ polyacrylamide gels using sodium dodecyl sulfate-PAGE and transferred onto nitrocellulose membranes. Membranes were incubated for $1 \mathrm{~h}$ at room temperature with Tris-buffered saline (2 mM Tris- $\mathrm{HCl}(\mathrm{pH} 8.0)$ and $15 \mathrm{mM} \mathrm{NaCl}(\mathrm{pH} 7.6)$ ) with $0.1 \%$ Tween 20 containing $5 \%$ nonfat dry milk powder to saturate nonspecific binding sites. Membranes were incubated with primary antibodies at the following dilutions: anti-human YAP1 antibody at 1:1000 (14074, Cell Signaling Technology), anti-human ESR2 antibody at 1:1000 (04-824, MerckMillipore), anti-human MMP2 antibody at 1:1000 (40994, Cell Signaling Technology), anti-human MMP9 antibody at $1: 1000$ (ab38898, abcam) and anti- $\beta$-actin antibody at $1: 1000$ (TA-09 and TA-08; ZSGB-BIO, China). All protein bands were quantified by densitometry using Image) software (National Institutes of Health, USA).

\section{Small-interfering RNA knockdown}

Primary ECSCs were cultured in growth medium as described previously to achieve approximately 70 to $80 \%$ confluence at the time of transfection. Transfection was performed using a small-interfering RNA (siRNA) (Life Technologies, Thermo Fisher Scientific) against YAP1: (F) 5'CAGCAGAAUAUGAUGAACUCGGCUU-3'; (R) 5'-AAGCCGAGUUCAUCAUAUUCUGCUG-3', ESR2: (F) 5'-CCUUUAGUGGUCCAUCGCCAGUUAU-3'; (R) 5'-AUAACUGGCGAUGGACCACUAAAGG-3' or a nontargeting negative control siRNA (low GC content; Life Technologies, Thermo Fisher Scientific) at a final concentration of $100 \mathrm{nM}$ using Lipofectamine RNAiMAX (Life Technologies, Thermo Fisher Scientific). The control group included non-treated cells. Cells were collected $48 \mathrm{~h}$ after transfection for RT-qPCR and Western blot analysis. 


\section{Plasmid overexpression}

ECSCs were cultured to approximately $80-90 \%$ confluence at the time of transfection. Lipofectamine 3000 (Life Technologies, Thermo Fisher Scientific) was used to transfect either empty pENTER plasmid or pENTER-YAP1 plasmid (CH862329, Vigene Biosciences, China) in Opti-MEM reduced-serum medium (Life Technologies, Thermo Fisher Scientific). The control group included non-treated cells. At $48 \mathrm{~h}$ post transfection, the cells were harvested and processed for RT-qPCR and Western blotting to verify overexpression efficiency and detect gene expression.

\section{Coimmunoprecipitation assay}

ECSCs were lysed with nondenaturing lysis buffer (Applygen Technologies, China) with $1 \%$ protease inhibitors and incubated on ice for $20 \mathrm{~min}$. The cell lysates were then centrifuged in a refrigerated microcentrifuge at $13,000 \mathrm{~g}$ for $20 \mathrm{~min}$ at $4^{\circ} \mathrm{C}$. The supernatants were used either directly for immunoprecipitation or stored at $80^{\circ} \mathrm{C}$. For immunoprecipitation, equal amounts of protein (500 g) were first immunoprecipitated with anti-YAP1 (14074, Cell Signaling Technology), anti-CHD4 (ab72418, abcam), or anti-MTA1 (ab71153, abcam) primary antibody at $4^{\circ} \mathrm{C}$ for 4 to $6 \mathrm{~h}$, followed by the addition of protein $\mathrm{A}$ agarose (11719408001, Roche) and incubation at $4^{\circ} \mathrm{C}$ overnight. The immunoprecipitates were collected by centrifugation, washed three times with PBS containing $0.5 \%$ protease inhibitors, and eluted with SDS-PAGE sample buffer. Immunoprecipitates were then analyzed by Western blotting as described previously.

\section{Chromatin immunoprecipitation and real-time qPCR}

Chromatin immunoprecipitation (ChIP) was performed using a ChIP assay kit (Pierce Chromatin Prep Module; Thermo Fisher Scientific) according to the manufacturer's instructions. Briefly, cells were crosslinked with 1\% formaldehyde for 10 min at room temperature and collected in PBS containing $1 \%$ protease inhibitors. The crosslinked cells were then lysed and enzymatically digested with micrococcal nuclease to shear genomic DNA. The following antibodies were used for immunoprecipitation: anti-YAP1 and polyclonal rabbit IgG. Protein/DNA complexes were eluted from the beads and treated with proteinase $\mathrm{K}$ solution at $65^{\circ} \mathrm{C}$ for $2.5 \mathrm{~h}$. RT-qPCR was performed on the purified DNA in the presence of SYBR Master Green Mix (Life Technologies, Thermo Fisher Scientific) using the ESR2 primers (F) 5'-aaaatgtgagtttcatgcctca-3'; (R) 5'-TCCAGGGGTAAGATGGATTG-3'; and 5'-ttgagcaatcacacctcagc-3'; (R) 5'-ctcacgcctgtaatcccaat-3' for amplification.

The $\Delta \Delta$ Ct method was used to analyze real-time ChIP results. Briefly, RT-qPCR was performed with a primer set targeting a positive locus and a primer set targeting a negative locus. Each ChIP DNA fraction's Ct value was normalized to the input DNA fraction Ct value for the same qPCR assay to account for differences in chromatin sample preparation: Ct(normalized $\mathrm{ChIP})=\mathrm{Ct}(\mathrm{ChIP})-\left(\mathrm{Ct}(\right.$ input $)-\log _{2}($ input dilution factor $\left.)\right)$. The difference between the ChIP fraction Ct values $(\Delta \Delta \mathrm{Ct})$ of the normalized experimental sample (S2) and the control sample (S1) was then determined: $\Delta \Delta \mathrm{Ct}(\mathrm{S} 2-\mathrm{S} 1)=\Delta \mathrm{Ct}(\mathrm{S} 2$ : normalized ChIP) $-\Delta \mathrm{Ct}(\mathrm{S} 1$ :normalized ChIP) Finally, the differential occupancy fold change (linear conversion of the second $\Delta \Delta \mathrm{Ct}$ to yield a fold change in site occupancy) was calculated: fold change in occupancy $=2\left({ }^{-\Delta \Delta \mathrm{Ct}(\mathrm{S} 2-\mathrm{S} 1)}\right)$.

\section{Matrigel invasion assay}

In vitro invasion assays were performed using Matrigel-coated (1:8 dilution) 24-well transwell chambers $(8-\mu \mathrm{m}$ pore size, $6.5-\mathrm{mm}$ diameter, Corning). Next, $2 \times 10^{5}$ primary, siRNAtransfected ECSCs were plated into the upper chambers in media without serum. The lower chambers were filled with $600 \mu \mathrm{L}$ of DMEM/F12 containing 20\% FBS. The cells were then incubated at $37^{\circ} \mathrm{C}$ for $48 \mathrm{~h}$. The cells on the upper surfaces of the filters together with the Matrigel were removed by wiping with a cotton swab. The inserts were then fixed in methanol for $30 \mathrm{~min}$ at room temperature and stained with hematoxylin. The stained cells reaching the lower surfaces of the transwells were observed and photographed using an Olympus DP71 microscope (Olympus). Five randomly selected fields were quantified for each experiment.

\section{Statistical analyses}

All experiments were performed at least three times. All data are presented as the mean and S.E.M. Comparisons of two groups were performed using the two-tailed Student's $t$-test. Comparisons of more than two groups were performed using one-way ANOVA. Statistical analyses were performed using the Statistical Package for the Social Science (SPSS) computer software version 22.0 (IBM SPSS Statistics). Figures were constructed using Prism 5 version 6.0f (GraphPad Software, Inc.). Power analyses were conducted using $\mathrm{G}^{*}$ Power version 3.1.9.6 based on the assumption of $\alpha=0.05$ (two-tailed). Powers $\geq 80 \%$ were considered statistically powered, and $P$-values $<0.05$ were considered statistically significant.

\section{Results}

\section{Differential expression of YAP1 in paired eutopic and ectopic endometrial tissues}

RT-qPCR and Western blotting results demonstrated differential expression of YAP1 in EU and EC tissues from the same patient with endometriomas. Paired endometriotic and endometrial tissues were used to measure YAP1 expression, and the results showed that YAP1 mRNA levels were significantly increased in the EU compared with the EC (5.9-fold; $n=20 ; P<0.01$; power=0.998; Fig. 1A). Western blotting showed that YAP1 protein expression was also significantly higher in the $\mathrm{EU}(n=20 ; P<0.05$; Fig. 1B).

\section{YAP1 functions as a transcriptional corepressor to suppress ESR2 expression in ECSCS}

As ESR2 plays a critical role in the pathological process of endometriosis, we asked whether YAP1, as a transcriptional coregulator, could regulate ESR2 expression. We used siRNAs to specifically knockdown YAP1 in ECSCs to measure the mRNA and protein levels 


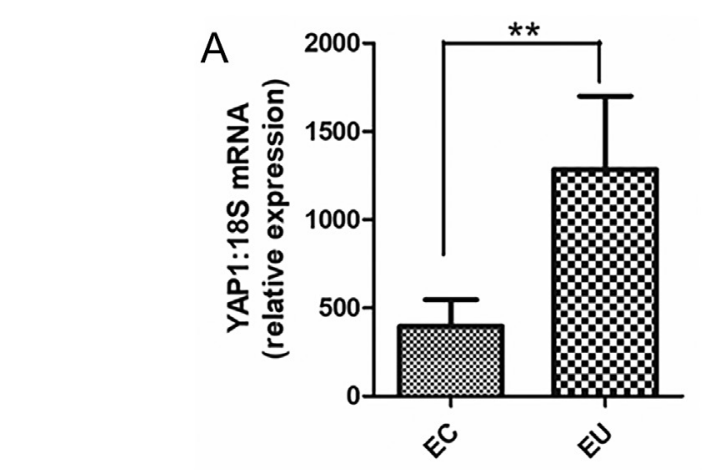

B
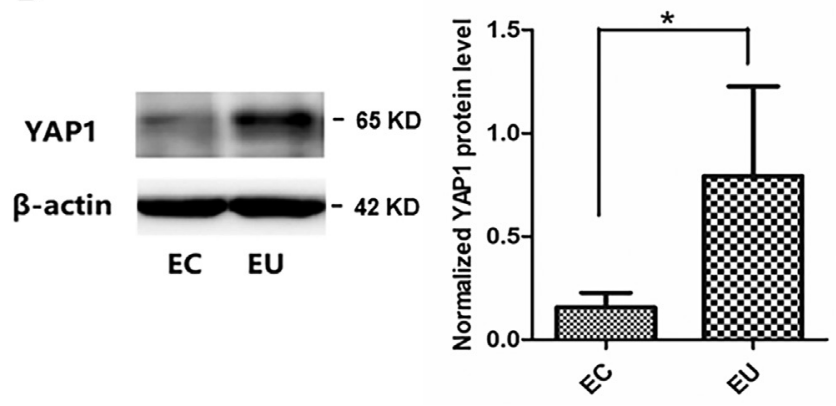

Figure 1 Differential expression of YAP1 in paired eutopic and ectopic endometrial tissues. Western blotting and quantification of YAP1 mRNA levels were performed as described. The values are the means \pm S.E.M. (A) YAP1 mRNA levels in paired eutopic endometrial tissues were higher than those in ectopic endometrial tissues $(n=20$; ${ }^{* *}, t$-test, $\left.P<0.01\right)$. (B) YAP1 protein expression in paired eutopic endometrial tissues was higher than that in ectopic endometrial tissues $\left(n=20 ;{ }^{*}, t\right.$-test, $\left.P<0.05\right)$. EU, eutopic endometrial tissues; EC, ectopic endometrial tissues. Error bars indicate \pm S.E.M. All experiments were repeated three times.

of ESR2. The control group included non-treated cells, and no significant difference was observed between the control group and the control siRNA group $(P>0.05)$. The efficacy of YAP1 siRNA knockdown was determined by both RT-qPCR and Western blotting (Fig. 2A). ECSCs transfected with siYAP1 exhibited diminished YAP1 $\mathrm{mRNA}$ and protein levels and significant increases in ESR2 mRNA levels (3.3-fold; $n=6 ; P<0.01$; power $=0.953$ ) and protein expression (Fig. 2B). Simultaneously, plasmid transfection was used to overexpress YAP1 in ECSCs (Fig. 2C), and no significant difference was observed between the control group and the control plasmid group $(P>0.05)$. The results showed that transfection of YAP1 overexpression plasmids suppressed ESR2 mRNA expression $(35 \% ; n=6 ; P<0.05 ;$ power $=0.804)$ and protein levels (Fig. 2D), indicating that YAP1 exerts an inhibitory effect on ESR2 expression in ECSCs.

\section{YAP1 directly binds to the promoter region of the ESR2 gene by recruiting the NuRD complex}

Previous studies have demonstrated that YAP1 exerts an inhibitory effect on gene expression by recruiting NuRD transcriptional repression complexes, particularly CHD4 and MTA1 (Kim et al. 2015). Thus, Co-IP assays were used to determine the interactions among YAP1, CHD4 and MTA1 in ECSCs. When YAP1 was immunoprecipitated from ECSCs, both CHD4 and MTA1 were present in the immunoprecipitate. Reciprocally, YAP1 was present in the CHD4 and MTA1 immunoprecipitates. Thus, we observed the formation of the YAP1-NuRD complex by YAP1, CHD4 and MTA1 ( $n=6$; Fig. $3 \mathrm{~A})$, showing that YAP1 could interact with the CHD4 and MTA1 proteins to form the YAP1-NuRD complex in ECSCs.

To confirm that YAP1 could bind to the promoter region of $E S R 2$ and participate in the regulation of ESR2 expression, we first examined the ESR2 gene sequence and found two possible YAP1 binding sequences (CATTCC) near the transcription initiation site of ESR2. ChIP assays were used to measure differences in the binding ability of YAP1 to ESR2 genes in paired eutopic and ectopic endometrial stromal cells. The results showed that YAP1 could bind to two sites, $(-539,-533)$ and $(-158,-152)$, upstream of the ESR2 transcription initiation site $(n=4)$. The binding of YAP1 to the two sites of the ESR2 promoter in ECSCs was significantly lower than that in EUSCs (4.44- and 3.43-fold, respectively; $n=4 ; P<0.01, \quad P<0.05$, respectively; power $=0.999$, power $=0.944$, respectively; Fig. $3 \mathrm{~B}$ ), suggesting that YAP1 might inhibit ESR2 expression by directly binding to its promoter region.

\section{YAP1 inhibits the invasiveness of ECSCs and the expression of MMP2 and MMP9 by suppressing ESR2 expression}

YAP1 has been shown to regulate cell invasion in different types of cancer cells, and a previous study confirmed that ESR2 can affect the invasive ability of endometriotic cells by regulating the expression of Snail (Han et al. 2015). To investigate whether YAP1 influences cell invasion by regulating ESR2 expression, we used siRNAs to knock down YAP1, ESR2 or both and measured changes in the mRNA and protein levels of MMP2 and MMP9, which are closely related to the invasiveness of ECSCs. The control group included nontreated cells, and no significant difference was observed between the control group and the control siRNA group $(P>0.05)$. The efficacy of siRNA knockdown was determined by RT-PCR and Western blotting $(n=6 ; P<0.01$; Fig. 4A). ECSCs transfected with siYAP1 exhibited 1.58-fold higher MMP2 mRNA levels that those of the controls, while ECSCs transfected with SiESR2 showed MMP2 mRNA levels that were markedly decreased by $35 \%$. However, MMP2 mRNA expression was reduced by $26 \%$ in cells transfected with SiYAP 1 and siESR2 together compared with that in cells transfected with siYAP1 alone $(n=6 ; P<0.01, P<0.01, P<0.01$, respectively; power $=0.998$; Fig. $4 \mathrm{~B}$ left). Likewise, the MMP9 mRNA levels in the siYAP1 group were 3.72-fold 



Figure 2 YAP1 functions as a transcriptional corepressor to suppress ESR2 expression in ECSCs. (A) SiRNA-mediated knockdown of YAP1 blocked YAP1 expression in ECSCs. ECSCs were serum-starved overnight and transfected with control siRNA or siYAP1. The control group included non-treated cells, and no significant difference was observed between the control group and the control siRNA group $(P>0.05)$. Cells were harvested for SYBR $®$ Green RT-qPCR and Western blotting $48 \mathrm{~h}$ after transfection for verification of siRNA knockdown efficiency. (B) SiRNAmediated knockdown of YAP1 increased ESR2 mRNA levels $\left(n=6 ;{ }^{* *} P<0.01\right)$ and ESR2 protein expression $(n=6)$. (C) ECSCs were transfected with the control plasmid or YAP1 plasmid for $48 \mathrm{~h}$. The control group included non-treated cells, and no significant difference
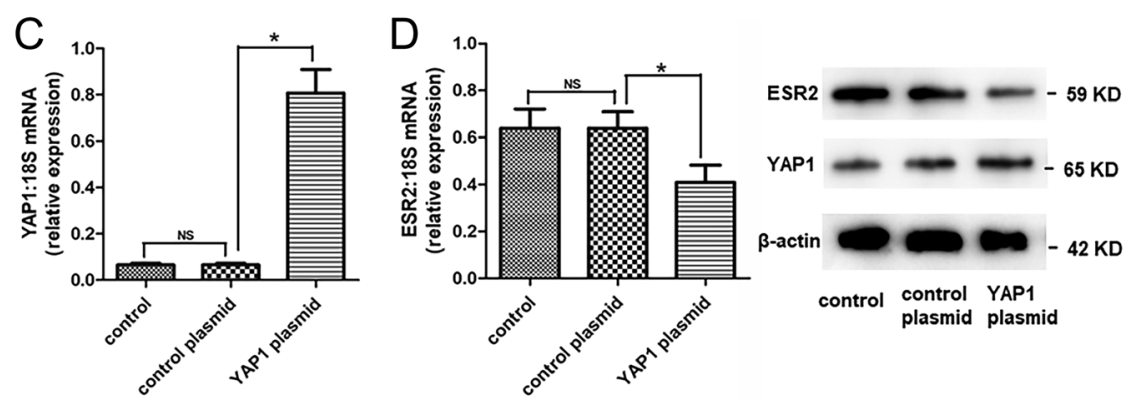
was observed between the control group and the control plasmid group $(P>0.05)$. Cells were then harvested for RT-PCR and Western blotting for verification of YAP1 overexpression efficiency. (D) Plasmid-mediated overexpression of YAP1 suppressed ESR2 mRNA $(n=6 ; * P<0.05)$ and ESR2 protein levels $(n=6)$. NS, not significant. Error bars indicate \pm S.E.M. All experiments were conducted in primary cultured cells and repeated three times.

higher than those in the control group, while the siESR2 group showed a 55\% decline in MMP9 mRNA levels. Compared with the SIYAP1 group, the SiYAP1 plus siESR2 group (SIYAP1+SiESR2) showed a $70 \%$ reduction $(n=6$; $P<0.05, P<0.05, P<0.05$, respectively; power $=0.921$; Fig. $4 \mathrm{~B}$ right). Similar results for MMP2 and MMP9 protein expression were observed, as shown in Fig. 4C. To further validate the role of ESR2 in YAP1 regulation of cell invasion, we conducted transwell experiments to evaluate invasion activity. ECSCs transfected with siYAP1 exhibited increased invasion activity $(n=5 ; P<0.05)$, while ECSCs transfected with siESR2 showed inhibited invasiveness $(n=5 ; P<0.05)$. However, transfection with siYAP1 and SiESR2 together decreased the number of invading cells compared with transfection with siYAP1 alone $(n=5 ; P<0.05$; Fig. 4D). Taken together, these findings indicated that YAP1 could inhibit the invasiveness of ECSCs and the expression of MMP2 and MMP9 by suppressing ESR2 expression.

\section{Discussion}

To our knowledge, this study provides the first direct evidence of the function of YAP1 in the regulation of $E S R 2$ and invasion of ovarian endometriosis. In this study, we showed that YAP1 expression was decreased in ectopic endometrium tissues from the cyst walls of ovarian endometriomas. YAP1 inhibited ESR2 expression by recruiting an NuRD transcriptional repression complex, which further downregulated invasion activity and MMP2 and MMP9 expression.

The role of YAP1 in cancer development remains controversial. Accumulative evidence suggests that the different roles of YAP1 in oncogenesis might be tissue- and cell context-specific. YAP1 overexpression in a wide variety of solid tumors, such as esophageal cancer, cervical cancer, hepatoma, and gastric cancer, and its association with a more aggressive tumor biology and worse survival strongly suggest that YAP1 plays oncogenic and anti-apoptotic roles in these tumor types (Song et al. 2015, Xiao et al. 2015, Cheng et al. 2016). However, YAP1 is defined as a tumor suppressor that induces apoptosis (Yuan et al. 2008, Bertini et al. 2009). Decreased or absent expression of YAP1 is highly correlated with tumor progression and worse survival for other tumors, such as breast cancer, colorectal cancer (CRC) and head and neck cancers (HNSCCs) (Wang et al. 2014). Inhibition of YAP1 in breast cancer cell lines suppresses breast cancer cell anoikis and increases cell migration and invasion (Tufail et al. 2012). Additionally, significantly decreased YAP1 expression has been noted in invasive carcinoma compared to normal breast tissues, suggesting that YAP1 acts as a tumor suppressor in invasive breast carcinomas (Cao et al. 2017). Furthermore, knockdown of YAP1 enhances cell proliferation, survival, migration, and resistance to cisplatin in HNSCC cell lines (Ehsanian et al. 2010). Moreover, YAP1 has also been reported to act as a tumor 


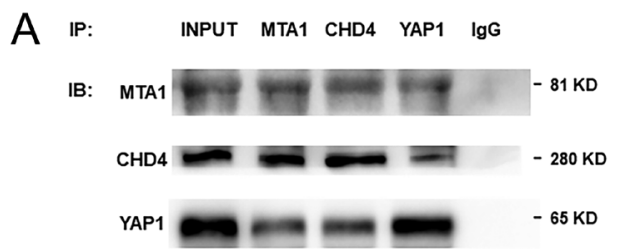

B
$(-539,-533)$

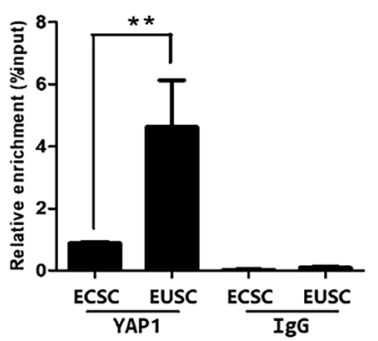

$(-158,-152)$

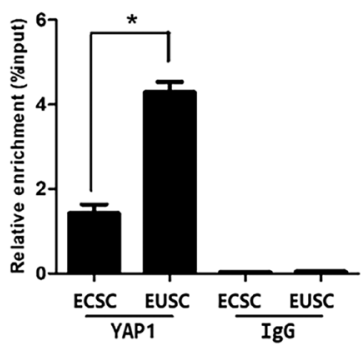

Figure 3 YAP1 binds to the ESR2 promotor region and inhibits its expression by recruiting the NuRD complex. (A) ECSC lysates were collected, immunoprecipitated using an anti-YAP1, anti-CHD4, anti-MTA1 or anti-IgG antibody, and analyzed by Western blotting with an anti-YAP1, anti-CHD4 or anti-MTA1 antibody. Formation of the YAP1-NuRD complex by YAP1, CHD4 and MTA1 was observed $(n=6)$. (B) YAP1 inhibits ESR2 expression by directly binding to its promoter region. Paired eutopic and ectopic endometrial stromal cells were harvested and subjected to ChIP using an anti-YAP1 or control IgG antibody, followed by SYBR® Green RT-qPCR. The binding of YAP1 to the two sites $(-539,-533)$ and $(-158,-152)$ upstream of the ESR2 promoter in ECSCs was significantly lower than that in EUSCs (4.44- and 3.43-fold, respectively; $n=4 ;{ }^{*} P<0.05$, **P<0.01). IB, immunoblot; IP, immunoprecipitation; EUSCs, eutopic endometrial stromal cells; ECSCs, ectopic endometrial stromal cells. All experiments were conducted in primary cultured cells and repeated three times.

suppressor in human CRC and restrict the growth of CRC xenografts (Barry et al. 2013). Consistent with the results of the previously mentioned studies, our study showed that YAP1 inhibits the expression of ESR2 and further suppresses cell invasion in ectopic stromal cells from ovarian endometriomas, thereby acting as a gene suppressor. The mechanism of YAP1's inhibitory effects on different cells is still not clear, and the specific mechanism of YAP1's action in cell suppression requires further exploration.

The correlation between YAP1 and the estradiol synthesis pathway has been studied in breast and ovarian granulosa cell carcinomas. Wang et al. discovered that YAP1 knockdown reduces FSH-induced aromatase protein expression and estrogen production in KGN cells, demonstrating that YAP1 plays an important role in regulating GCT cell proliferation, migration and steroidogenesis (Fu et al. 2014). Guan et al. proved that verteporfin, which disrupts the interaction between YAP1/ TAZ and TEADs, inhibits breast cancer cell proliferation and migration and tumor growth (Zhou et al. 2015). Endometriosis is an estrogen-dependent disease, and ectopic lesions grow in the presence of estrogen (Bulun
2009, Xiong et al. 2015). Previous researchers and our team have found that aromatase, the key rate-limiting enzyme that catalyzes the conversion of androgens to estrogens, is highly expressed in the ECSCs of ovarian endometriomas (Bulun et al. 2005, Zeng et al. 2015). The important role of ESR2 in ovarian endometriosis has also been confirmed. Previous studies have shown that epigenetic ESR2 promoter regulation can account for high ESR2 levels and that ESR2 is also responsible for low ERS1 expression in ECSCs (Xue et al. 2007). Increased expression of ESR2 inhibits the transcriptional activity of ESR 1 in endometriosis, resulting in a decrease in the expression level of the progesterone receptor $(P G R)$ (Bulun et al. 2010). Additionally, a positive role for ESR2 in endometriotic stromal cell cycle progression and proliferation has been proposed (Trukhacheva et al. 2009). Recently, O'Malley et al. (Han et al. 2015) proposed that the SRC-1 isoform/ESR2 complex can be a next-generation endometriosis therapeutic target. Thus far, the relationship between YAP1 and ESR2 has not been studied. Our study is the first to show that YAP1 regulates ESR2 transcription and ESR2 translation. SiRNA knockdown of YAP1 increases ESR2 mRNA expression and protein levels in ECSCs of ovarian endometriomas and that plasmid overexpression of YAP1 significantly decreases ESR2 expression. Our study proposes that YAP1 might be a protective factor in ovarian endometriosis and provides new evidence for the role of YAP1 in suppressing gene expression.

As a transcriptional coactivator, YAP1 can bind to oncogenes or tumor suppressor gene transcription factors, the most important of which are the TEAD transcription factors. Knockdown of TEADs or disruption of the YAP1-TEAD complex abolishes YAP1-dependent gene transcription and diminishes YAP1-induced cell proliferation, oncogenic transformation, and the epithelial-to-mesenchymal transition (EMT). Lamar et al. found that YAP1 promotes the metastasis of breast cancer and melanoma cells and that the TEAD-interacting domain of YAP1 is essential for YAP1-mediated tumor growth and metastasis (Lamar et al. 2012). However, studies have shown that YAP1 not only promotes gene transcription but also regulates gene expression. Mechanistically, the YAP1/TAZ-TEAD complex recruits the NuRD complex to deacetylate histones and alters nucleosome occupancy at target genes. NuRD is a complex comprising many submits, including CHD4, MTA1 and GATAD2A. The NuRD complex has been proposed to mediate the repressor activity of the YAP1/TAZ-TEAD-Smad3 complex in human ES cells (Beyer et al. 2013). Lim et al. generalized the role of the YAP1/TAZ-TEAD-NuRD complex in the regulation of cell growth and survival, which might be the major function of YAP1/TAZ (Kim et al. 2015). Furthermore, the results of our Co-IP analysis showed that YAP1 interacts with both CHD4 and MTA1, while YAP1 knockdown or overexpression affects the binding of CHD4 and MTA1 

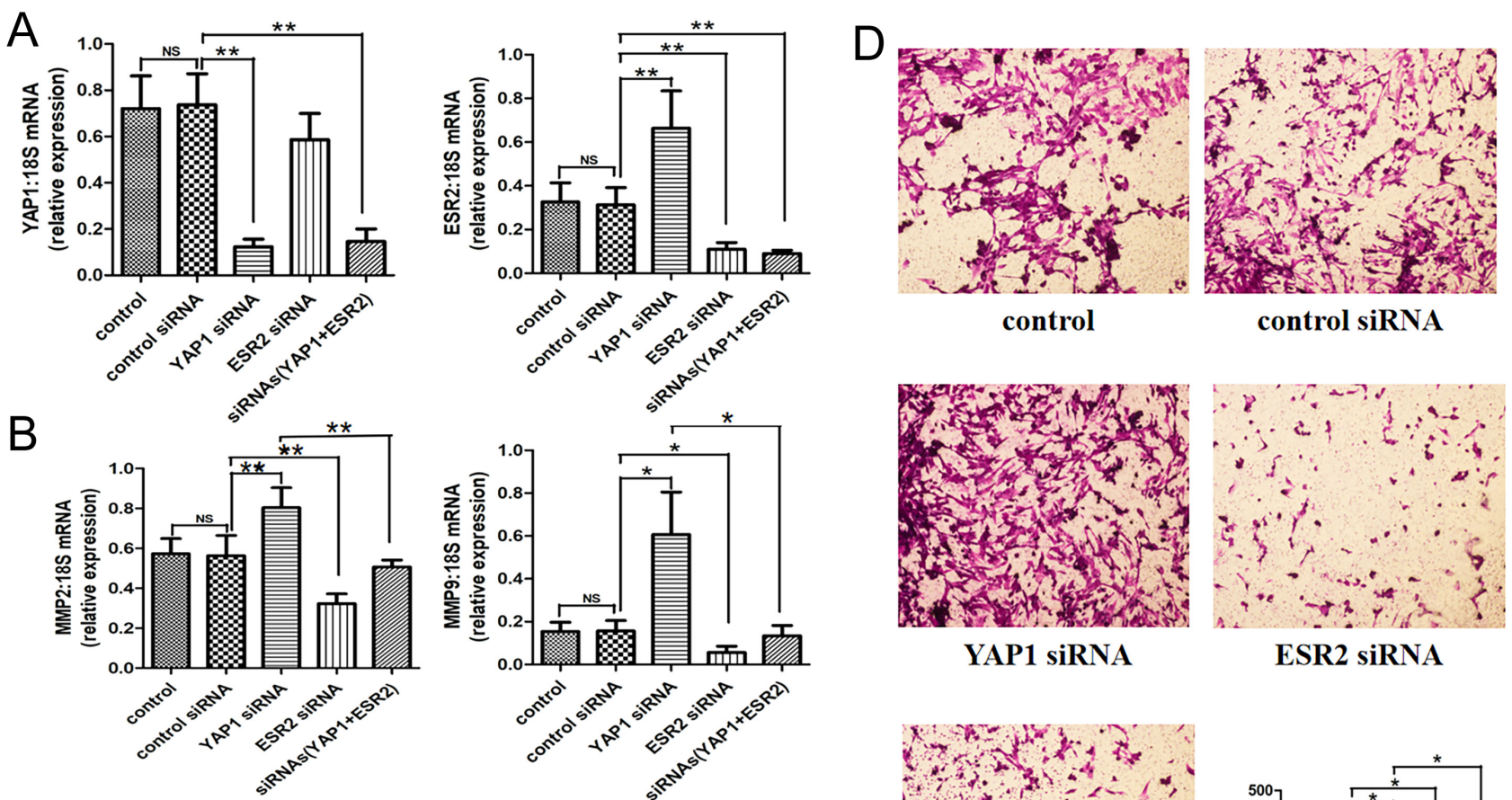

YAP1 siRNA

ESR2 siRNA
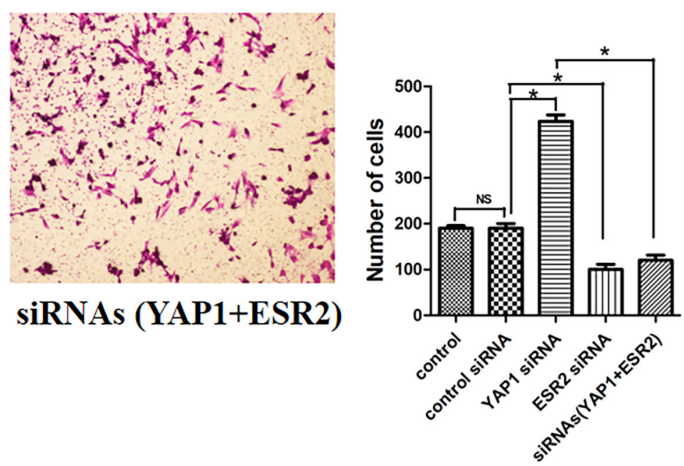

Figure 4 YAP1 inhibits ECSC invasion and MMP2 and MMP9 expression by suppressing the expression of ESR2. (A) ECSCs were controltransfected or transfected with the indicated siRNAs and serum-starved overnight. The control group included non-treated cells, and no significant difference was observed between the control group and the control siRNA group $(P>0.05)$. Cells were harvested for RT-PCR and Western blotting with anti-YAP1 and anti-ESR2 antibodies for verification of siRNA knockout efficiency $\left(n=6\right.$; $\left.{ }^{* *} P<0.01\right)$. (B) ECSCs transfected with siYAP1 alone exhibited increased MMP2 and MMP9 mRNA levels compared to the controls (1.58-fold, 3.72-fold, respectively; $n=6$; $\left.* P<0.05,{ }^{*} P<0.01\right)$, while ECSCs transfected with siESR2 alone showed decreased MMP2 and MMP9 mRNA levels $(35 \%$, 55\%, respectively; $n=6$; $\left.{ }^{*} P<0.05,{ }^{*} P<0.01\right) . M M P 2$ and MMP9 mRNA levels were reduced in cells transfected with siYAP1 and siESR2 together compared with those in cell transfected with siYAP1 alone $(26 \%, 70 \%$, respectively; $n=6 ; * P<0.05, * * P<0.01)$. Cells were harvested for SYBR Green RT-qPCR. (C) Similar results for MMP2 and MMP9 protein expression were observed. Likewise, ECSCs transfected with siYAP1 alone exhibited increased MMP2 and MMP9 protein expression compared with the controls, while ECSCs transfected with siESR2 alone showed decreased MMP2 and MMP9 protein expression. MMP2 and MMP9 protein expression levels were reduced in cells transfected with siYAP1 and siESR2 together compared with those in cells transfected with siYAP1 alone $(n=6 ; P<0.05)$. Cells were harvested for Western blotting. (D) To further validate the role of ESR2 in YAP1 regulation of cell invasion, a transwell assay was conducted to evaluate invasion activity. ECSCs transfected with SIYAP1 alone exhibited increased invasion activity, while ECSCs transfected with siESR2 alone showed inhibited invasiveness. Meanwhile, the invasiveness of ECSCs was reduced in cells transfected with SIYAP1 and SIESR2 together compared with that of cells transfected with siYAP1 alone $(n=5 ; * P<0.05)$. Cells were harvested for SYBR $B$ Green RT-qPCR and Western blotting. NS, not significant. Error bars indicate \pm S.E.M. All experiments were conducted in primary cultured cells and repeated three times.

to target genes for cell growth and survival. Consistent with previous studies, our study demonstrated that YAP1 first interacted with the CHD4 and MTA1 proteins and then formed a YAP1-NuRD complex to regulate ESR2 expression. Whether this complex can regulate key enzymes involved in estrogen synthesis and related pathways in endometriosis warrants further study.
Knockdown of YAP1 enhances the proliferation, migration, and invasion of cancer cells, and restoration of YAP1 expression in these knockdown cells reduces the tumorigenic properties of the cells (Wang et al. 2014). MMPs are important proteases that degrade the extracellular matrix and thus promote tumor metastasis and invasion. YAP1 is highly expressed in human lung 
adenocarcinomas (LACs) compared to normal lung tissue (NLT), and knockdown of YAP1 may inhibit the proliferation and invasion of adenocarcinoma cells through downregulation of MMP9 (Cui et al. 2012). The YAP1 pathway is required for LATS1-induced inhibition of cell growth and invasion, and LATS1 restrains the nuclear transfer of YAP1 and downregulates YAP1, MMP2 and MMP9 expression (Cheng et al. 2016). Furthermore, O'Malley et al. (Han et al. 2015) discovered that ESR2 enhances invasion activity in human endometriotic epithelial cells (iHEECs). However, whether YAP1 can influence cell invasion by regulating ESR2 expression has not been discussed. In our study, we found that knockdown of ESR2 reduced invasion activity by ECSCs and decreased the expression of MMP2 and MMP9, further confirming the regulation of endometriosis invasion by ESR2. Moreover, ECSCs transfected with SiYAP1 exhibit increased MMP2 and MMP9 levels and enhanced invasion activity. However, invasion activity and MMP2 and MMP9 expression were reduced in ECSCs transfected with siYAP1 and siESR2 together compared to that in cells transfected with siYAP1 alone. These results demonstrate that YAP1 can inhibit invasion activity and MMPs expression by inhibiting ESR2 expression in ECSCs of ovarian endometriomas, representing a new potential therapeutic target for the treatment of endometriosis.

Recently, several studies highlighted the essential role of YAP1 in the pathogenesis of endometriosis involving different endometriotic lesions. Pei and colleagues investigated the relationship between the YAP1 pathway and cell autophagy in endometriosis (Pei et al. 2019) and found that the mRNA expression of YAP1 was increased in the EUSCs of endometriosis patients compared with healthy controls with no statistically significant difference and that the protein levels were significantly increased in the EUSCs. In this study, our results demonstrated that decreased expression of YAP1 in ovarian endometriotic lesions is correlated with high ESR2 expression and cell invasion, which is consistent with studies in breast cancer cell lines (Tufail et al. 2012). However, Lin et al. (2017) showed that YAP1 expression in the EC tissues of patients with endometriosis was higher than that in the $\mathrm{EU}$ of normal control patients, suggesting a progressive role for YAP1 in the development of endometriosis. Different tissue origins and cell- or tissue-specific activities should be considered for the opposite results. EC tissues from patients with endometriosis and normal EU tissues from normal participants were used as study models in their study, while the differences in YAP1 expression in our study were detected using paired EC tissues from ovarian endometriomas and $\mathrm{EU}$ tissues from the same patient. Moreover, we BLAST searched YAP1 primers from both papers, and the primer that we used included all eight transcripts formed by the YAP1 gene (Gaffney et al. 2012) and was tested by other studies (Konsavage et al. 2012, Hayashi et al. 2015), but the previously mentioned study detected five of eight transcripts of YAP1. Different transcripts may act as transcriptional repressors and activators, which may lead to dual functions for YAP1 in promoting and inhibiting cell growth in human cancers. Therefore, YAP1 expression and function in endometriosis still require further exploration. A major limitation of this study is the lack of experiments in vivo with an endometriosis mouse model, which may further validate the therapeutic effect of YAP1 in the pathogenesis of endometriosis. Whether YAP1 regulates the expression of other receptors, such as ESR1 and PGR, warrants further exploration. Additionally, the regulation of YAP1 alongside other important pathogenic factors of endometriosis, such as PGE2 and VEGF, is worth investigating.

\section{Conclusion}

In summary, our study demonstrates that decreased levels of YAP1 in ovarian endometriotic tissues enhance ESR2 expression via formation of a YAP1-NuRD complex, which further binds to the ESR2 promotors. Furthermore, YAP1 inhibits ovarian ESC invasion and MMP2 and MMP9 expression. Alterations in this YAP1/ESR2mediated regulation of ovarian endometriosis cell invasion may represent a potential mechanism for women with endometriosis. Further research is needed to improve our understanding of this regulation and to identify new molecular targets for interventions and treatments for endometriosis.

\section{Declaration of interest}

The authors declare that there is no conflict of interest that could be perceived as prejudicing the impartiality of the research reported.

\section{Funding}

This work was supported by the National Key R\&D Program of China (Grant number 2017YFC1001200), the National Natural Science Foundation of China (Grant number 81671427) and Beijing Natural Science Foundation (Grant number 7194325).

\section{Author contribution statement}

C Z, Z-T D and Q X conceived the study and wrote the paper. C Z, P-L W and Z-T D performed the experiments and analyzed the data. $L X$ and $Y-F Z$ collected samples.

\section{Acknowledgements}

The authors thank Prof Ding-Fang Bu and Doctor Xue-Ying Li for generous advice regarding this study. 


\section{References}

Barry ER, Morikawa T, Butler BL, Shrestha K, De La Rosa R, Yan KS, Fuchs CS, Magness ST, Smits R, Ogino S et al. 2013 Restriction of intestinal stem cell expansion and the regenerative response by YAP. Nature 493 106-110. (https://doi.org/10.1038/nature11693)

Bertini E, Oka T, Sudol M, Strano S \& Blandino G 2009 YAP: at the crossroad between transformation and tumor suppression. Cell Cycle $\mathbf{8}$ 49-57. (https://doi.org/10.4161/cc.8.1.7259)

Beyer TA, Weiss A, Khomchuk Y, Huang K, Ogunjimi AA, Varelas X \& Wrana JL 2013 Switch enhancers interpret TGF-beta and Hippo signaling to control cell fate in human embryonic stem cells. Cell Reports $\mathbf{5}$ 1611-1624. (https://doi.org/10.1016/j.celrep.2013.11.021)

Bulun SE 2009 Endometriosis. New England Journal of Medicine 360 268-279. (https://doi.org/10.1056/NEJMra0804690)

Bulun SE, Lin Z, Imir G, Amin S, Demura M, Yilmaz B, Martin R, Utsunomiya H, Thung S, Gurates B et al. 2005 Regulation of aromatase expression in estrogen-responsive breast and uterine disease: from bench to treatment. Pharmacological Reviews 57 359-383. (https://doi. org/10.1124/pr.57.3.6)

Bulun SE, Cheng YH, Pavone ME, Xue Q, Attar E, Trukhacheva E, Tokunaga H, Utsunomiya H, Yin P, Luo X et al. 2010 Estrogen receptor-beta, estrogen receptor-alpha, and progesterone resistance in endometriosis. Seminars in Reproductive Medicine 28 36-43. (https:// doi.org/10.1055/s-0029-1242991)

Camargo FD, Gokhale S, Johnnidis JB, Fu D, Bell GW, Jaenisch R \& Brummelkamp TR 2007 YAP1 increases organ size and expands undifferentiated progenitor cells. Current Biology 17 2054-2060. (https://doi.org/10.1016/j.cub.2007.10.039)

Cao L, Sun PL, Yao M, Jia M \& Gao H 2017 Expression of YESassociated protein (YAP) and its clinical significance in breast cancer tissues. Human Pathology 68 166-174. (https://doi.org/10.1016/j. humpath.2017.08.032)

Cheng $H$, Zhang $Z$, Rodriguez-Barrueco R, Borczuk A, Liu $H, Y u$ Silva JM, Cheng SK, Perez-Soler R \& Halmos B 2016 Functional genomics screen identifies YAP1 as a key determinant to enhance treatment sensitivity in lung cancer cells. Oncotarget 7 28976-28988. (https://doi.org/10.18632/oncotarget.6721)

Cui ZL, Han FF, Peng XH, Chen X, Luan CY, Han RC, Xu WG \& Guo XJ 2012 YES-associated protein 1 promotes adenocarcinoma growth and metastasis through activation of the receptor tyrosine kinase Axl. International Journal of Immunopathology and Pharmacology 25 989-1001. (https://doi.org/10.1177/039463201202500416)

Dong J, Feldmann G, Huang J, Wu S, Zhang N, Comerford SA, Gayyed MF, Anders RA, Maitra A \& Pan D 2007 Elucidation of a universal sizecontrol mechanism in Drosophila and mammals. Cell 130 1120-1133. (https://doi.org/10.1016/j.cell.2007.07.019)

Ehsanian R, Brown M, Lu H, Yang XP, Pattatheyil A, Yan B, Duggal P, Chuang R, Doondeea J, Feller S et al. 2010 YAP dysregulation by phosphorylation or DeltaNp63-mediated gene repression promotes proliferation, survival and migration in head and neck cancer subsets. Oncogene 29 6160-6171. (https://doi.org/10.1038/onc.2010.339)

Fu D, Lv X, Hua G, He C, Dong J, Lele SM, Li DW, Zhai Q, Davis JS \& Wang C 2014 YAP regulates cell proliferation, migration, and steroidogenesis in adult granulosa cell tumors. Endocrine-Related Cancer 21 297-310. (https://doi.org/10.1530/ERC-13-0339)

Gaffney CJ, Oka T, Mazack V, Hilman D, Gat U, Muramatsu T, Inazawa J, Golden A, Carey DJ, Farooq A et al. 2012 Identification, basic characterization and evolutionary analysis of differentially spliced mRNA isoforms of human YAP1 gene. Gene 509 215-222. (https://doi. org/10.1016/j.gene.2012.08.025)

Greene AD, Lang SA, Kendziorski JA, Sroga-Rios JM, Herzog TJ \& Burns KA 2016 Endometriosis: where are we and where are we going? Reproduction 152 R63-R78. (https://doi.org/10.1530/REP-16-0052)

Han SJ, Jung SY, Wu SP, Hawkins SM, Park MJ, Kyo S, Qin J, Lydon JP, Tsai SY, Tsai MJ et al. 2015 Estrogen receptor beta modulates apoptosis complexes and the inflammasome to drive the pathogenesis of endometriosis. Cell 163 960-974. (https://doi.org/10.1016/j. cell.2015.10.034)

Hayashi H, Higashi T, Yokoyama N, Kaida T, Sakamoto K, Fukushima Y, Ishimoto T, Kuroki H, Nitta H, Hashimoto D et al. 2015 An imbalance in TAZ and YAP expression in hepatocellular carcinoma confers cancer stem cell-like behaviors contributing to disease progression. Cancer Research 75 4985-4997. (https://doi.org/10.1158/0008-5472.CAN-150291)

Jarzabek K, Koda M, Walentowicz-Sadlecka M, Grabiec M, Laudanski P \& Wolczynski S 2013 Altered expression of ERs, aromatase, and COX2 connected to estrogen action in type 1 endometrial cancer biology. Tumour Biology 34 4007-4016. (https://doi.org/10.1007/s13277-0130991-9)

Kim M, Kim T, Johnson RL \& Lim DS 2015 Transcriptional co-repressor function of the hippo pathway transducers YAP and TAZ. Cell Reports 11 270-282. (https://doi.org/10.1016/j.celrep.2015.03.015)

Konsavage WM, Kyler SL, Rennoll SA, Jin G \& Yochum GS 2012 Wnt/betacatenin signaling regulates Yes-associated protein (YAP) gene expression in colorectal carcinoma cells. Journal of Biological Chemistry 287 11730-11739. (https://doi.org/10.1074/jbc.M111.327767)

Lamar JM, Stern P, Liu H, Schindler JW, Jiang ZG \& Hynes RO 2012 The Hippo pathway target, YAP, promotes metastasis through its TEADinteraction domain. PNAS 109 E2441-E2450. (https://doi.org/10.1073/ pnas.1212021109)

Lin SC, Lee HC, Hou PC, Fu JL, Wu MH \& Tsai SJ 2017 Targeting hypoxiamediated YAP1 nuclear translocation ameliorates pathogenesis of endometriosis without compromising maternal fertility. Journal of Pathology 242 476-487. (https://doi.org/10.1002/path.4922)

Liu H, Zhang Z, Xiong W, Zhang L, Xiong Y, Li N, He H, du Y \& Liu Y 2017 Hypoxia-inducible factor-1alpha promotes endometrial stromal cells migration and invasion by upregulating autophagy in endometriosis. Reproduction 153 809-820. (https://doi.org/10.1530/REP-16-0643)

Maia H, Haddad C, Coelho G \& Casoy J 2012 Role of inflammation and aromatase expression in the eutopic endometrium and its relationship with the development of endometriosis. Women's Health 8 647-658. (https://doi.org/10.2217/whe.12.52)

Mo JS, Park HW \& Guan KL 2014 The Hippo signaling pathway in stem cell biology and cancer. EMBO Reports 15 642-656. (https://doi. org/10.15252/embr.201438638)

Pan D 2010 The hippo signaling pathway in development and cancer. Developmental Cell 19 491-505. (https://doi.org/10.1016/j. devcel.2010.09.011)

Pei T, Huang X, Long Y, Duan C, Liu T, Li Y \& Huang W 2019 Increased expression of YAP is associated with decreased cell autophagy in the eutopic endometrial stromal cells of endometriosis. Molecular and Cellular Endocrinology 491 110432. (https://doi.org/10.1016/j. mce.2019.04.012)

Ryan IP \& Taylor RN 1997 Endometriosis and infertility: new concepts. Obstetrical and Gynecological Survey 52 365-371. (https://doi. org/10.1097/00006254-199706000-00021)

Ryan IP, Schriock ED \& Taylor RN 1994 Isolation, characterization, and comparison of human endometrial and endometriosis cells in vitro. Journal of Clinical Endocrinology and Metabolism 78 642-649. (https:// doi.org/10.1210/jcem.78.3.8126136)

Song S, Honjo S, Jin J, Chang SS, Scott AW, Chen Q, Kalhor N, Correa AM, Hofstetter WL, Albarracin CT et al. 2015 The hippo coactivator YAP1 mediates EGFR overexpression and confers chemoresistance in esophageal cancer. Clinical Cancer Research 21 2580-2590. (https:// doi.org/10.1158/1078-0432.CCR-14-2191)

Trukhacheva E, Lin Z, Reierstad S, Cheng YH, Milad M \& Bulun SE 2009 Estrogen receptor (ER) beta regulates ERalpha expression in stromal cells derived from ovarian endometriosis. Journal of Clinical Endocrinology and Metabolism 94 615-622. (https://doi.org/10.1210/jc.2008-1466)

Tufail R, Jorda M, Zhao W, Reis I \& Nawaz Z 2012 Loss of Yes-associated protein (YAP) expression is associated with estrogen and progesterone receptors negativity in invasive breast carcinomas. Breast Cancer Research and Treatment 131 743-750. (https://doi.org/10.1007/s10549011-1435-0)

Vercellini P, Vigano P, Somigliana E \& Fedele L 2014 Endometriosis: pathogenesis and treatment. Nature Reviews: Endocrinology 10 261-275. (https://doi.org/10.1038/nrendo.2013.255)

Wang H, Du YC, Zhou XJ, Liu H \& Tang SC 2014 The dual functions of YAP-1 to promote and inhibit cell growth in human malignancy. Cancer Metastasis Reviews 33 173-181. (https://doi.org/10.1007/s10555-0139463-3)

Xiao H, Jiang N, Zhou B, Liu Q \& du C 2015 TAZ regulates cell proliferation and epithelial-mesenchymal transition of human hepatocellular 
carcinoma. Cancer Science 106 151-159. (https://doi.org/10.1111/ cas.12587)

Xiong W, Zhang L, Yu L, Xie W, Man Y, Xiong Y, Liu H \& Liu Y 2015 Estradiol promotes cells invasion by activating beta-catenin signaling pathway in endometriosis. Reproduction 150 507-516. (https://doi. org/10.1530/REP-15-0371)

Xiu-Li W, Wen-Jun C, Hui-Hua D, Su-Ping H \& Shi-Long F 2009 ERB-041, a selective ER beta agonist, inhibits iNOS production in LPS-activated peritoneal macrophages of endometriosis via suppression of NFkappaB activation. Molecular Immunology 46 2413-2418. (https://doi. org/10.1016/j.molimm.2009.04.014)

Xue Q, Lin Z, Cheng YH, Huang CC, Marsh E, Yin P, Milad MP, Confino E, Reierstad S, Innes J et al. 2007 Promoter methylation regulates estrogen receptor 2 in human endometrium and endometriosis. Biology of Reproduction 77 681-687. (https://doi.org/10.1095/ biolreprod.107.061804)

Yuan M, Tomlinson V, Lara R, Holliday D, Chelala C, Harada T, Gangeswaran R, Manson-Bishop C, Smith P, Danovi SA et al. 2008 Yes-associated protein (YAP) functions as a tumor suppressor in breast. Cell Death and Differentiation 15 1752-1759. (https://doi.org/10.1038/ cdd.2008.108)
Zanconato F, Cordenonsi M \& Piccolo S 2016 YAP/TAZ at the roots of cancer. Cancer Cell 29 783-803. (https://doi.org/10.1016/j.ccell.2016.05.005)

Zeng C, Xu JN, Zhou Y, Yang HX, Zhou YF \& Xue Q 2015 C-Jun $\mathrm{NH} 2$-terminal kinase and p38 inhibition suppresses prostaglandin E2-stimulated aromatase and estrogen receptor levels in human endometriosis. Journal of Clinical Endocrinology and Metabolism 100 E1404-E1414. (https://doi.org/10.1210/jc.2015-2031)

Zhou X, Wang S, Wang Z, Feng X, Liu P, Lv XB, Li F, Yu FX, Sun Y, Yuan H et al. 2015 Estrogen regulates Hippo signaling via GPER in breast cancer. Journal of Clinical Investigation 125 2123-2135. (https://doi. org/10.1172/JCl79573)

Received 23 November 2019

First decision 15 January 2020

Revised manuscript received 10 May 2020

Accepted 15 June 2020 